\title{
A Maneuvering Flight Noise Model for Helicopter Mission Planning
}

\author{
Eric Greenwood \\ Research Aerospace Engineer \\ NASA Langley Research Center \\ Hampton, VA
}

\author{
Robert Rau \\ USRP Intern \\ NASA Langley Research Center \\ Hampton, VA
}

\author{
Benjamin May \\ Senior Quality Engineer \\ Wyle \\ Arlington, VA
}

\author{
Christopher Hobbs \\ Senior Acoustician \\ Wyle \\ Arlington, VA
}

\begin{abstract}
A new model for estimating the noise radiation during maneuvering flight is developed in this paper. The model applies the Quasi-Static Acoustic Mapping (Q-SAM) method to a database of acoustic spheres generated using the Fundamental Rotorcraft Acoustics Modeling from Experiments (FRAME) technique. A method is developed to generate a realistic flight trajectory from a limited set of waypoints and is used to calculate the quasi-static operating condition and corresponding acoustic sphere for the vehicle throughout the maneuver. By using a previously computed database of acoustic spheres, the acoustic impact of proposed helicopter operations can be rapidly predicted for use in mission-planning. The resulting FRAME-QS model is applied to near-horizon noise measurements collected for the Bell 430 helicopter undergoing transient pitch up and roll maneuvers, with good agreement between the measured data and the FRAME-QS model.
\end{abstract}

\section{NOTATION}

a Inertial frame acceleration.

$a_{l o n} \quad$ Longitudinal acceleration.

$C_{T} \quad$ Main rotor thrust coefficient.

$C_{W} \quad$ Main rotor weight coefficient.

$D \quad$ Fuselage parasite drag.

$f(t) \quad$ Cubic spline trajectory function.

$g \quad$ Gravitational acceleration.

$M_{H} \quad$ Main rotor hover tip Mach number.

$n_{a} \quad$ "Inertial tip-path-plane" normal vector.

$n_{z} \quad$ Load factor.

$t \quad$ Time.

$v \quad$ Inertial frame velocity.

$V \quad$ Speed.

$W \quad$ Weight.

$x \quad$ Inertial frame position.

$\alpha \quad$ Rotor tip-path-plane angle of attack.

$\gamma \quad$ Flight path angle.

$\gamma_{e} \quad$ Effective flight path angle.

$\rho \quad$ Spline smoothing parameter.

$\mu \quad$ Main rotor advance ratio.

$\theta \quad$ Main rotor tip-path-plane pitch angle.

$\phi \quad$ Main rotor tip-path-plane roll angle.

\section{OBJECTIVE}

The objective of this paper is to develop a model for helicopter harmonic noise radiation that is suitable for use in planning realistic helicopter operations for both civil and military operators.

Presented at the American Helicopter Society 71th Annual Forum, Virginia Beach, VA, May 5-7, 2015. This is a work of the U.S. Government and is not subject to copyright protection in the U.S.
In order to be useful, this model must accurately predict the dominant harmonic noise radiation of both the main and tail rotor:

1. over the entire range of operating conditions typically flown by the helicopter, including transient maneuvers at varying load factors;

2. in all radiation directions, including those not normally measured during steady straight-line flight and;

3. be computationally efficient, to enable multiple iterations for the timely development of low noise flight strategies.

\section{INTRODUCTION}

Helicopter operators are increasingly interested in the acoustic impact of their operations. For military operators, detection of helicopters by hostile forces is often triggered by acoustic emissions, making acoustics a key element of mission survivability. For civil operators, helicopter noise drives community annoyance of helicopter operations resulting in complaints. In response, local and national governments are increasingly pressured to limit helicopter operations in noise sensitive communities. The negative impacts of helicopter noise can be mitigated by incorporating acoustic analysis into mission planning tools, allowing operators to design missions that reduce acoustic impacts while still effectively completing the mission objectives. While several empirical models are currently used for acoustic mission planning, they are limited to modeling the helicopter noise source in steady-state flight conditions. In practice, helicopter operations consist of both steady state and maneuvering flight conditions. During maneuvering flight, the noise radiation characteristics of the helicopter can change dramatically, causing both annoyance and triggering acoustic detection (Ref. 1). 
The Rotorcraft Noise Model (RNM), developed by Wyle Laboratories and NASA, is one of the more widely used models for evaluating the acoustic impacts of helicopter operations (Refs. 2-4). An upgraded version of the model was developed by Wyle, and is now called the Advanced Acoustic Model (AAM) (Ref. 5). The user can input a series of waypoints defining the helicopter's planned trajectory. The model subdivides this trajectory into a number of steady-state flight segments, each of which is then associated with an acoustic sphere representing the frequency and magnitude of noise radiated by the helicopter over a range of directions for that particular steady flight condition. RNM (and AAM) will not compute the change in orientation of the steady-state flight acoustic spheres due to maneuvering flight; however, the user can manually specify an orientation for each segment if it is known through other means.

In practice, acoustic spheres for each steady flight condition are constructed from ground-based microphone measurements of helicopter noise during steady straight-line flight by a process called the Acoustic Repropagation Technique (ART). A noise measurement technique improving on the ART concept was later developed by researchers at the German Aerospace Center (DLR) that allows helicopter noise radiation to be characterized for maneuvering flight conditions by using a distributed array of ground-based microphones to capture a large range of radiation directions over a short period of time (Refs. 6,7). However, routine application of the technique is difficult due to both the number of microphones and the large number of test points required to fully characterize the helicopter over the entire envelope of feasible maneuvers.

The Quasi-Static Acoustic Mapping (Q-SAM) method, developed by Gopalan and Schmitz at University of Maryland, permits a first-order equivalence to be made between the acoustic state of steady flight conditions and accelerating or decelerating flight conditions (Refs. 8,9). The effectiveness of the technique was demonstrated in several flight test campaigns (Refs. 10,11). Later, the method was extended to steady turning flight, but in the absence of data at different rotor thrust levels, applicability of the technique is limited to small increases $(<10 \%)$ in the load factor (Ref. 12). Q-SAM was integrated into RNM (RNM-QS) allowing a set of steady straight-line flight noise spheres to be generalized to a range of moderate accelerating and decelerating straight-line flight conditions.

The Fundamental Rotorcraft Acoustic Modeling from Experiments (FRAME) technique, developed by the first author, constructs non-dimensional aeroacoustic models of rotor noise radiation by fitting analytical models to measured acoustic data from both wind tunnel and flight experiments. Because an analytical model of the major rotor harmonic noise sources is constructed, noise estimates can be obtained at flight conditions and radiation directions that were not originally measured (Refs. 13,14). The FRAME model was later extended to maneuvering flight conditions by introducing a dynamic prescribed wake model for Blade-Vortex Interaction (BVI) noise (Ref. 15). The model showed good agreement with measured data for several transient maneuvers and could be run in real time for a single observer; however, the generation of ground noise contours for mission planning typically requires noise calculations for hundreds to thousands of observers-far too many for the model to be practically applied in the design and evaluation of low noise mission profiles.

Recently, Sickenberger (Ref. 16) compared the results of first-principles noise predictions of a maneuvering helicopter with a dynamic (i.e. distorted) wake model and a quasi-static wake model. Only minor differences were noted between the noise emitted by the two models, even during very aggressive longitudinal maneuvers. Similarly, no significant differences were observed between the noise radiation predicted when using dynamic and quasi-static rotor blade flapping models. Sickenberger also demonstrated that, in addition to changes in the aerodynamic state of the rotor, a major cause of the large increases in noise radiation observed during transient maneuvering flight was due to the re-orientation of the rotor tip-path-plane with respect to observers on the ground. For example, observers in the plane of the horizon can be exposed to high noise levels radiated out of the plane of the rotor. It was also shown that the noise radiated above the rotor plane is poorly approximated by the noise radiated below the plane of the rotor, such as that measured by ground-based microphones during acoustic flight testing.

These findings suggest that the Q-SAM method could be applied to more aggressive maneuvers, allowing a limited set of quasi-static acoustic data to be used to model a wide range of transient maneuvers. However, in order for Q-SAM to be applied to such aggressive maneuvers, data must be obtained for noise radiation at the appropriate load factors and in directions (with respect to the rotor tip-path-plane) not normally observed during steady flight.

\section{APPROACH}

The approach developed in this paper is to combine the strengths of the RNM-QS and FRAME methods, resulting in a noise model that can accurately estimate helicopter harmonic noise radiation during maneuvering flight in a computationally efficient manner. FRAME is used to generalize measured data from steady-flight noise measurements to a range of load factors. Because FRAME builds a computational model from the data, far-field noise levels can be estimated over all directions with respect to the main rotor hub, and normalized to a fixed distance, producing a full-sphere of noise data suitable for use in mission planning tools like RNM. By repeating the process for operating conditions spanning the practical range of advance ratios, $\mu$, thrust coefficients, $C_{T}$, and main rotor tip-path-plane angles of attack, $\alpha$, a database of noise spheres can be generated in advance and rapidly applied to predict the acoustic state of the helicopter at a later time.

Given a set of noise data for steady flight conditions, the Q-SAM principle can then be applied to quasi-statically select the appropriate noise spheres by determining to first-order the operating state of the helicopter at each point along the maneuvering flight trajectory. Users of mission planning tools 
typically define the flight trajectory using a minimal set of waypoints. However, in order to estimate the flight condition of the helicopter throughout the trajectory, including transient maneuvers, a higher resolution trajectory is required. One effective way of generating smooth and realistic trajectories commonly used in other applications is to fit a minimum acceleration smoothing spline that passes near each of the user-defined waypoints (Ref. 17). In this paper, a cubic smoothing spline describing the trajectory of the helicopter in all three dimensions as a function of time, $\vec{f}(t)$, is developed from the waypoints so as to minimize the expression:

$$
\rho \sum_{i=1}^{N}\left|\vec{f}\left(t_{i}\right)-\vec{x}_{i}\right|^{2}+\int_{t_{1}}^{t_{N}}\left|\frac{d^{2}}{d t^{2}} \vec{f}(t)\right|^{2} d t
$$

where $t_{1}$ and $t_{N}$ are the time of the first and last waypoint of the trajectory, $\vec{x}_{i}$ are the Cartesian coordinates of the $i^{\text {th }}$ waypoint, and smoothing parameter $\rho$ controls the relative weighting of closeness of fit (for large values of $\rho$ ) to smoothness (for small values of $\rho$ ) (Ref. 18). The spline fit is solved iteratively, varying $\rho$ in order to determine the smoothest fit (i.e. minimum acceleration) such that the generated trajectory is no further than a specified distance away from the user-supplied waypoints $\vec{x}_{i}$.

Having now calculated a smooth and continuous cubic spline trajectory as a function of time, the three-dimensional accelerations of the helicopter can be obtained. Because the spline is made up of piece-wise cubic polynomials, the expressions for the velocity and acceleration along the spline trajectory are exact and guaranteed to be smooth; numerical differentiation is not required. Given the accelerations along the path in all three dimensions, the net accelerations on the vehicle can be computed in the inertial reference frame as:

$$
\vec{a}(t)=a_{x}(t) \hat{i}+a_{y}(t) \hat{j}+\left(a_{z}(t)+g\right) \hat{k}
$$

The load factor, $n_{z}$, in the vehicle body reference frame can then be approximated as the magnitude of the acceleration vector normalized by gravity, i.e. $n_{z}=|\vec{a}(t)| / g$, under the assumptions that the vehicle can be represented as a point mass and that the aerodynamic forces imparted on the fuselage are much less than the inertial forces, including gravity. Likewise, assuming that the drag of the fuselage, for a given airspeed $V$, does not change much as a function of angle of attack or sideslip, then the change in the rotor thrust vector is solely dependent on inertial accelerations such that:

$$
\hat{n}_{a}(t)=\frac{\vec{a}(t)}{|\vec{a}(t)|}
$$

These assumptions may be violated for maneuvers that are not well coordinated, e.g. when the helicopter enters a highly sideslipped flight condition.

Making the typical assumption that the rotor tip-path-plane is normal to the thrust vector, $\hat{n}_{a}$ can also be used to described the change in rotor tip-path-plane orientation in the inertial reference frame. In accordance with the Q-SAM equivalence, the acoustic state of the helicopter is assumed equivalent if the rotor tip-path-plane angle of attack is the same, in addition to the airspeed, RPM, and load factor. For moderate accelerations, the tip-path-plane angle of attack can be calculated to firstorder (Ref. 8):

$$
\alpha=-\frac{D}{W}-\gamma-\frac{a_{l o n}}{g}
$$

When the airspeed, $V$, is held constant this results in the QSAM effective flight path angle for moderate longitudinal accelerations (Ref. 9):

$$
\gamma_{e}=\gamma+\frac{a_{l o n}}{g}
$$

which relates the change in tip-path-plane angle of attack due to longitudinal acceleration, $a_{l o n} / g$, to that due to change in the flight path angle, $\gamma$.

Likewise, for transient maneuvers in all three dimensions, the effective flight path angle, $\gamma_{e}$, can be viewed as the angle between the inertial velocity vector computed from the smoothing spline, $\vec{v}$, and the change in the tip-path-plane orientation due to inertial acceleration, described by the inertial tip-pathplane unit normal vector $\hat{n}_{a}$. Therefore, the effective flight path angle corresponding to an equivalent rotor tip-path-plane angle of attack during transient maneuvering flight can be determined using the following expression:

$$
\sin \gamma_{e}=\frac{\vec{v} \cdot \hat{n}_{a}}{V}
$$

Consequently, when the helicopter is in steady flight, $\hat{n}_{a}$ is aligned with gravity in the inertial axes, and the expected result $\gamma_{e}=\gamma$ is obtained.

Given the effective flight path angle, speed, and load factor, the appropriate FRAME sphere can be looked up quasistatically from the database and rotated with the calculated change in rotor tip-path-plane orientation, $\hat{n}_{a}$. First, the attitude of the helicopter is defined to be the projection of the inertial velocity vector onto the inertial tip-path-plane:

$$
\hat{u}=\frac{\vec{v}-\left(\vec{v} \cdot \hat{n}_{a}\right) \hat{n}_{a}}{V}
$$

Then, the change in the pitch angle is determined as:

$$
\sin \Delta \theta=u_{z}
$$

Finally, the change in the roll angle is found with the following expression:

$$
\tan \Delta \phi=-\frac{(\hat{k} \times \hat{u}) \cdot \hat{n}_{a}}{\hat{n}_{a} \cdot(\hat{u} \times \hat{k} \times \hat{u})}
$$

This paper focuses on source noise modeling, so noise is propagated from the sphere to observers on the ground using spherical spreading and the model applied only over short propagation ranges; however, the method is compatible with the more sophisticated long-range propagation models used by some mission planning tools. An outline of the resulting FRAME-QS method is shown in Figure 1. 


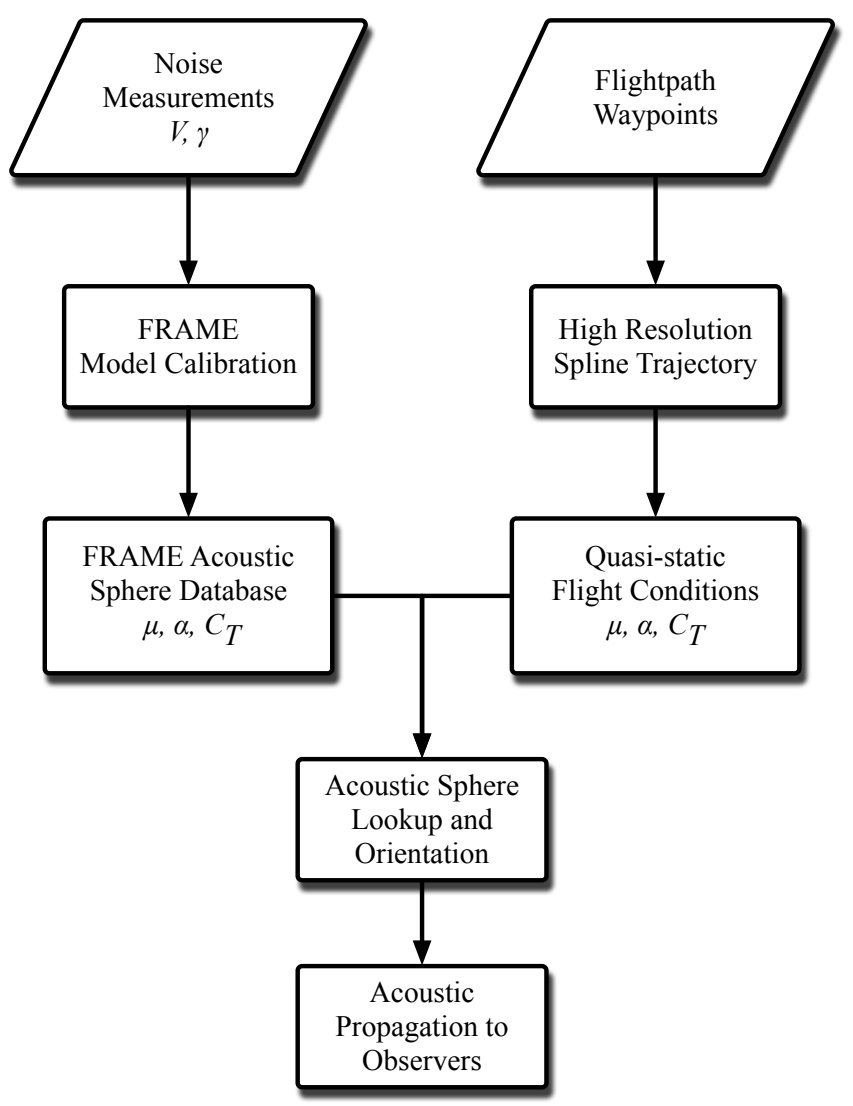

Fig. 1. Flowchart of the FRAME-QS method.

\section{VALIDATION}

In a joint test program, NASA, Bell Helicopter, and the US Army collected a large database of noise measurements for the Bell 430 helicopter at Eglin Air Force Base in 2011. This unique data set includes acoustics measurements of transient maneuvers such as pitch-ups, push-overs, rolls, longitudinal accelerations and decelerations, and combinations of these maneuvers over a distributed array of microphones. Additional measurements were collected using specialized arrays for noise characterization during steady-state flight conditions and for the development of ground-noise contours for a variety of possible landing approach paths. For all three types of noise measurement, vehicle inertial and aerodynamic data were measured and synchronized in time to the acoustic recordings. The test set up and collected data are described in extensive detail by Watts et al. (Refs. 19,20) The FRAME-QS model introduced in this paper was validated against two aggressive maneuvers selected from this dataset: a $1.5 \mathrm{~g}$ pitch-up maneuver from a $6^{\circ}$ descent and a $10^{\circ} \%$ roll towards the advancing side of the helicopter from level flight.

The FRAME modeling parameters were calibrated to measured noise data using three steady-state flight conditions: $130 \mathrm{kts}$ level flight, $80 \mathrm{kts}$ level flight, and $70 \mathrm{kts}$ descending flight along a $9^{\circ}$ flight path angle. The model expands upon the previously developed and validated FRAME model of the Bell 430 helicopter (Ref. 21) in that the thickness and lower-harmonic loading noise radiated by the tail rotor are included in the model, using the same approach as the main rotor noise prediction except that the distorted wake model is replaced with a simplified linear inflow model. FRAME was applied to compute a database of nearly 500 predicted acoustic spheres covering airspeeds from $40 \mathrm{kts}$ to $130 \mathrm{kts}$, flight path angles of $3^{\circ}$ (climb) to $-18^{\circ}$ (descent), and load factors from 0.75 to 2.0. Spheres for intermediate conditions not in the database are computed as needed using natural neighbor interpolation (Ref. 22) over the three-dimensional $\left(V, \gamma, n_{z}\right)$ Delaunay triagulation between the conditions for which spheres do exist in the database.

Figure 2 shows the Overall Sound Pressure Level (OASPL) noise contours predicted by FRAME over the surface of an acoustic sphere for a $100 \mathrm{kts}$ level flight condition. The OASPL metric emphasizes the lower harmonic thickness and loading noise radiated by the main rotor. Figure $2 \mathrm{a}$ shows a Lambert projection of the noise radiated above the horizon, where the perimeter of the plot shows the noise radiated in the plane of the horizon from azimuths of $0^{\circ}$ (behind the helicopter), through $90^{\circ}$ on the advancing side, to $180^{\circ}$ ahead of the helicopter, and elevation angles from $0^{\circ}$ (in the horizon) to $90^{\circ}$ (directly above the helicopter). Similarly, Figure $2 b$ shows the predicted noise radiation below the horizon plane, from elevation angles of $0^{\circ}$ to $-90^{\circ}$ (directly below the helicopter). It is clear from the estimated noise level contours that the magnitude and directivity of the lower-harmonic noise radiation on the upper hemisphere is different than that of the lower hemisphere, consistent with the findings of Sickenberger in Reference 16. This suggests that models which assume noise radiation to be symmetric about the rotor tip-path-plane may lead to inaccurate results when these radiation directions are oriented towards observers on the ground, such as during a steeply banked turn. However, no data is currently availible with which to validate these above-the-plane noise predictions.

FRAME acoustic sphere A-Weighted Sound Pressure Level (A-SPL) noise contours for a $100 \mathrm{kts} 7.5^{\circ}$ descending flight condition are shown in Figure 3. For this flight condition, BVI noise dominates the A-SPL metric in most directions, although tail rotor thickness noise also contributes in the plane of the tail rotor. Both the upper and lower halves of the acoustic sphere are plotted in Figures 3a and 3b, respectively. The upper and lower A-SPL contours are symmetric about the main rotor tip-path-plane - this is because the BVI noise, modeled by dipole noise sources on the rotor blades, radiates with equal amplitude above and below the rotor disk, although the phase of the BVI impulses is reversed. In contrast, the main rotor lower harmonic noise is caused by both thickness (monopole) and loading (dipole) noise sources; the coherent addition of the two resulting in the significant dissymmetry across the rotor tip-path-plane shown in Figure 2.

The acoustic hemispheres were applied to predict noise using three different models of increasing complexity:

1. The "Conventional" model is representative of the source noise models used in existing mission planning tools. The predicted flight track is composed of linear segments between each user-selected waypoint. Acoustic spheres 


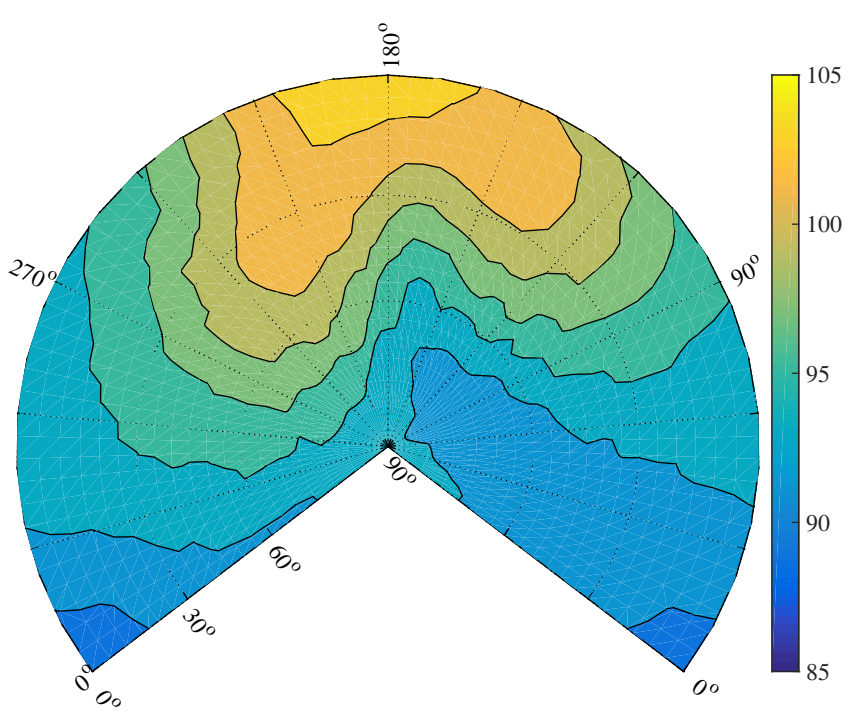

(a) Upper Hemisphere. dB OASPL.

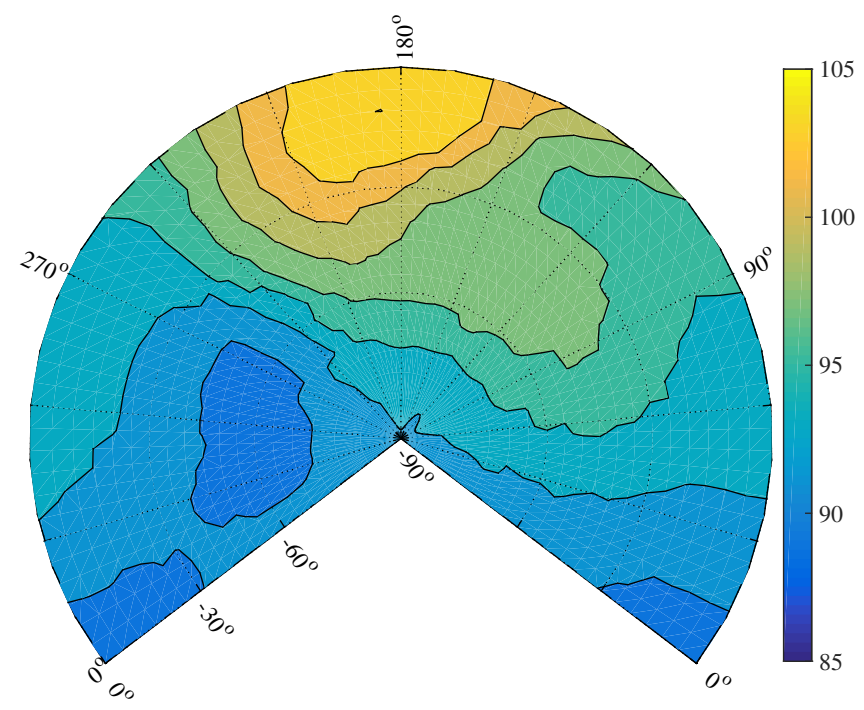

(b) Lower Hemisphere. dB OASPL.

Fig. 2. Predicted FRAME acoustic sphere for $100 \mathrm{kts}$ level flight.

are selected along each segment from the subset of the database where the load factor, $n_{z}$, is equal to one, using the flight path angle of the segment, $\gamma$, and the current velocity, $V$, under the assumption of constant acceleration between waypoints. The steady-state spheres are oriented with the horizon.

2. The FRAME-QS model with $C_{T}=C_{W}$ uses a predicted flight track following the high resolution spline trajectory described previously. The trajectory is used to estimate the three dimensional acceleration of the helicopter, determining both the change in the sphere orientation relative to steady flight and the effective flight path angle, following the Q-SAM equivalence. Spheres are selected along the flight track from the subset of the database where $n_{z}=1$ based on the velocity, $V$, and effective flight path angle, $\gamma_{e}$, every $1 / 10^{\text {th }}$ of a second.

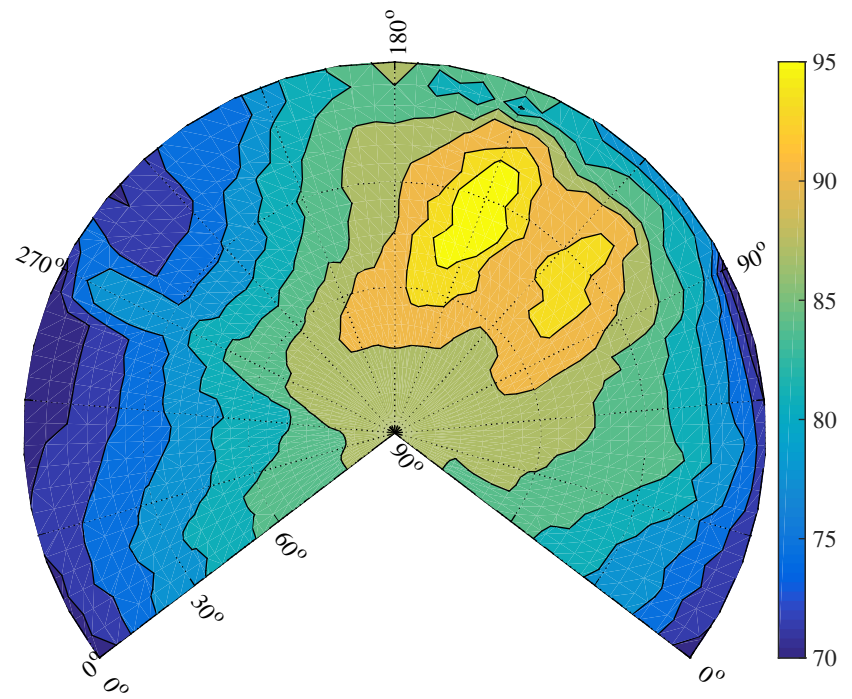

(a) Upper Hemisphere. dB A-Weighted SPL.

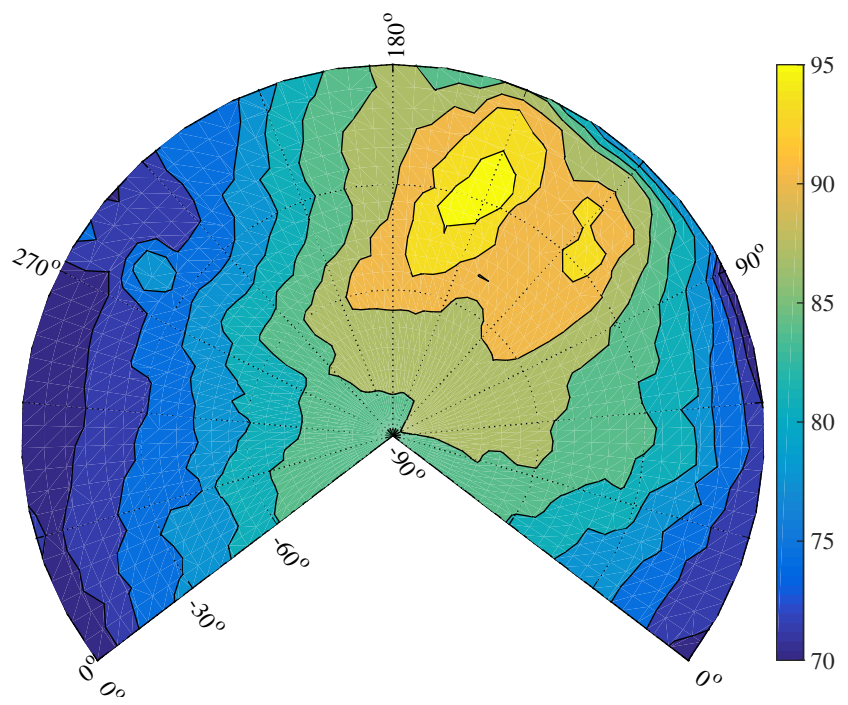

(b) Lower Hemisphere. dB A-Weighted SPL.

Fig. 3. Predicted FRAME acoustic sphere for 100 kts $7.5^{\circ}$ descending flight.

3. The FRAME-QS model with variable $C_{T}$ works in the same manner as the FRAME-QS model with constant $C_{T}$, except that spheres are selected from the entire database that includes variations in the current load factor, $n_{z}$, in addition to velocity, $V$, and effective flight path angle, $\gamma_{e}$.

\section{Fast Pitch Up from Descending Flight}

The first maneuver examined was an aggressive pitch up (nearly $10^{\circ} \mathrm{s}^{-1}$ ) initiated from a steady $6^{\circ}$ descent at $80 \mathrm{kts}$. During the maneuver, the helicopter's load factor reached a maximum value of $1.5 \mathrm{~g}$. The maneuver was initiated from an altitude of about 330 feet, approximately 1,500 feet directly ahead of the reference microphone located on the nominal flight track. In addition to the reference microphone location, predictions are compared to measurements made at microphones 850 feet to both the advancing and retreating sides of 
the flight track. These locations correspond to observation angles at elevations approximately $10^{\circ}$ below the horizon and at azimuths directly ahead of the helicopter and $30^{\circ}$ to either side of the nose upon the initiation of the pitch up maneuver.

A high resolution trajectory for the FRAME-QS models was generated from a small set of waypoints using the minimal acceleration smoothing spline approach. Figure 4 shows the measured and predicted vertical trajectory of the helicopter over the transient portion of the maneuver. The smoothing spline trajectory matches the actual path of the helicopter quite closely. The simple dynamics model does a good job estimating the helicopter's state from this trajectory throughout the maneuver; for example, Figure 5 plots the estimated pitch rate of the helicopter against that measured by the on board inertial measurement system mounted in the helicopter's fuselage. The rotor tip-path-plane was shown to maintain its orientation with respect to the fuselage within $1^{\circ}$ throughout the maneuver using an optical tip-path-plane tracking system (Ref. 23) developed by the University of Maryland.

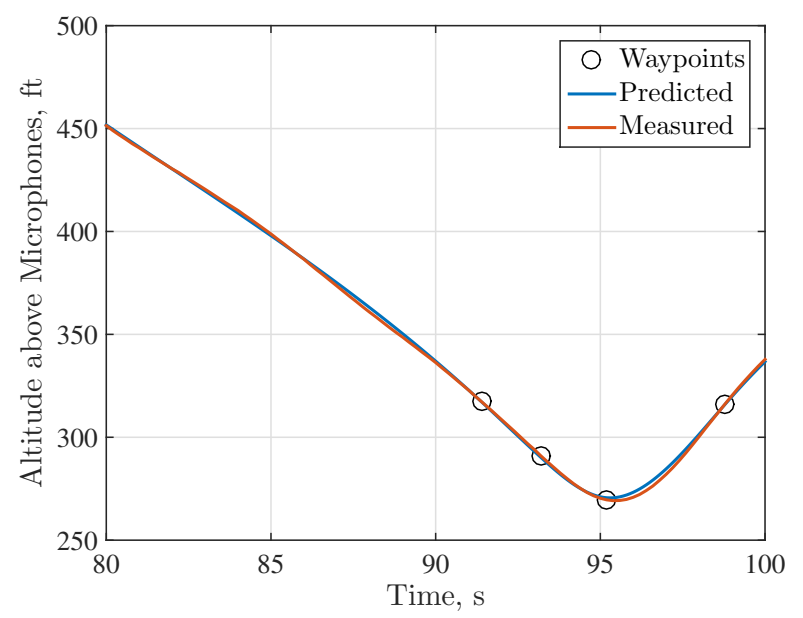

Fig. 4. Vertical trajectory during the pitch up maneuver.

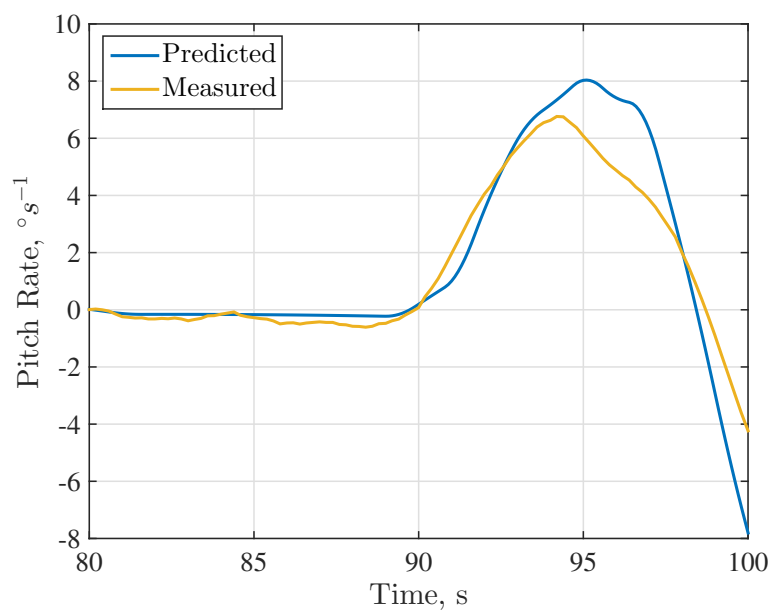

Fig. 5. Pitch rate during pitch up maneuver.
Figure 6 shows the predicted OASPL time histories for the three levels of modeling, as compared to the measured values at each microphone location. The OASPL values are computed for the measured data using a quarter second time interval (about five main rotor blade passages) to show the transient nature of the noise during the maneuver. All noise levels have been normalized to a distance of 10 rotor radii from the main rotor hub in order to emphasize the effect of the choice of source noise model on the predicted levels. At all three observer locations, the measured lower harmonic noise increases in response to the maneuver (initiated about $91 \mathrm{~s}$ after the start of the "run"). The FRAME-QS model with variable $C_{T}$ also demonstrates this behavior, capturing the transient increase in noise within $2 \mathrm{~dB}$ in all three directions. The FRAME-QS model with constant load factor $\left(C_{T}=C_{W}\right)$ shows some change in OASPL due to the transient maneuver as the orientation of the acoustic hemisphere varies in response to the acceleration of the helicopter along the flight trajectory; however, the large increase in noise levels due to the increased load factor of the helicopter is not accurately predicted when thrust is held constant. This is most likely because the increased load factor results in an increase in the lower harmonic loading noise radiated out to observers near the horizon as the helicopter pitches up. The peak difference in noise levels between the two FRAME-QS approaches is similar to what would be estimated by scaling the noise by the load factor $\left(n_{z}\right)$ using the heuristic $20 \log _{10} n_{z} \approx 3.5 \mathrm{~dB}$. The "Conventional" model does not show any increase due to the transient pitch up maneuver, with the normalized noise levels gradually decreasing as the helicopter approaches the array and the observers move farther out of the plane of the horizon.

Figure 7 shows the same comparison, but using the AWeighted SPL metric to emphasize noise relevant to annoyance, such as BVI and the tail rotor. Once again, the measured noise levels are seen to increase in response to the transient maneuver. A "double-bang" of two separate peaks in the noise level is seen on all three microphones, consistent with the BVI noise reported on by Sickenberger et al. (Ref. 1) for the pitch up maneuver of the Bell 206 helicopter and later modeled using FRAME with a dynamic prescribed wake model in Reference 15. The first peak of the "double-bang" is caused by the rotor wake initially passing through the tip-path-plane shortly after initiation of the pitch up, reducing the vortex miss-distance and increasing BVI noise. Continued deceleration causes the wake to move above the rotor plane, decreasing BVI, followed by the second BVI peak as the deceleration slows and the wake passes back through the rotor.

The FRAME-QS model with variable $C_{T}$ captures the "double-bang" BVI phenomena, achieving similar peak BVI levels as seen in the measured data; however, in the model the first BVI peak is louder than the second for the centerline observer whereas the opposite is true for the measured data. The "double-bang" is also captured by the FRAME-QS model with constant load factor, although the predicted peak levels are less representative of the measured data. For the centerline and retreating side observers, the constant load factor model tends to underpredict noise levels, similar to lower harmonic 

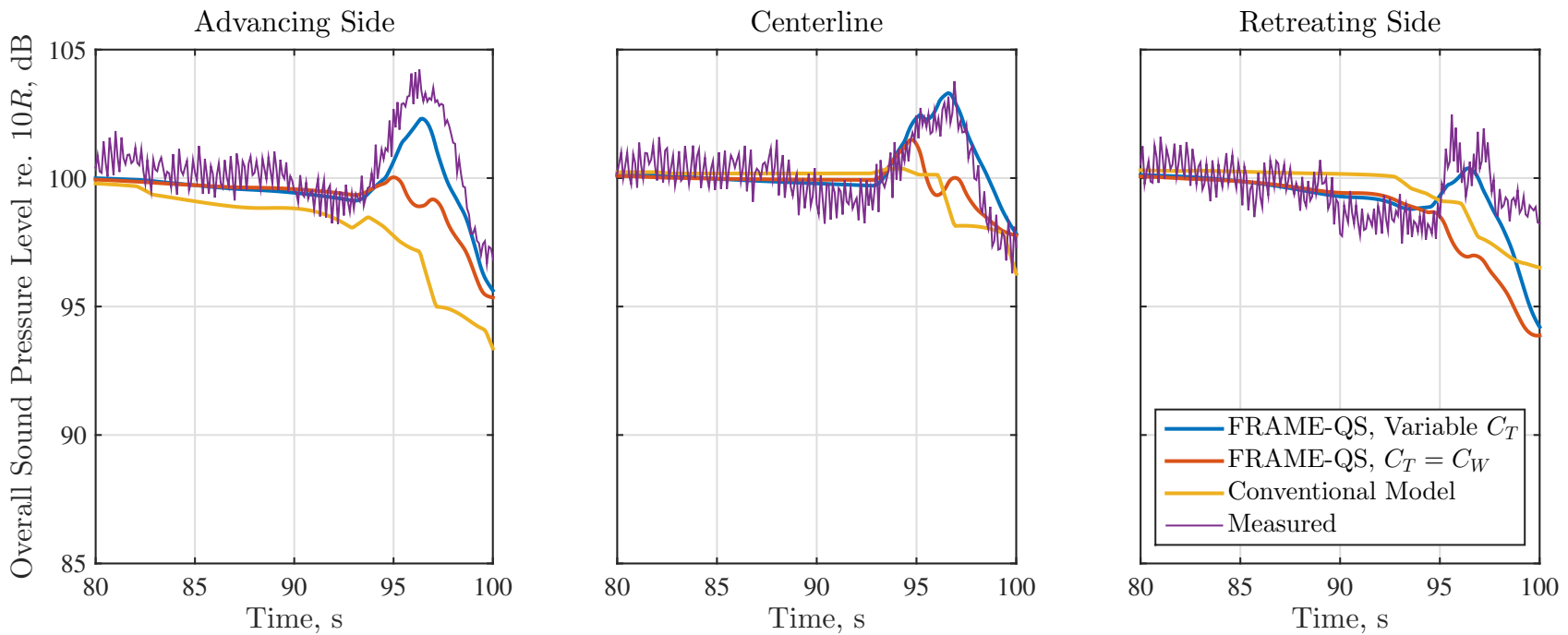

Fig. 6. Overall Sound Pressure Levels for the fast pitch up maneuver.
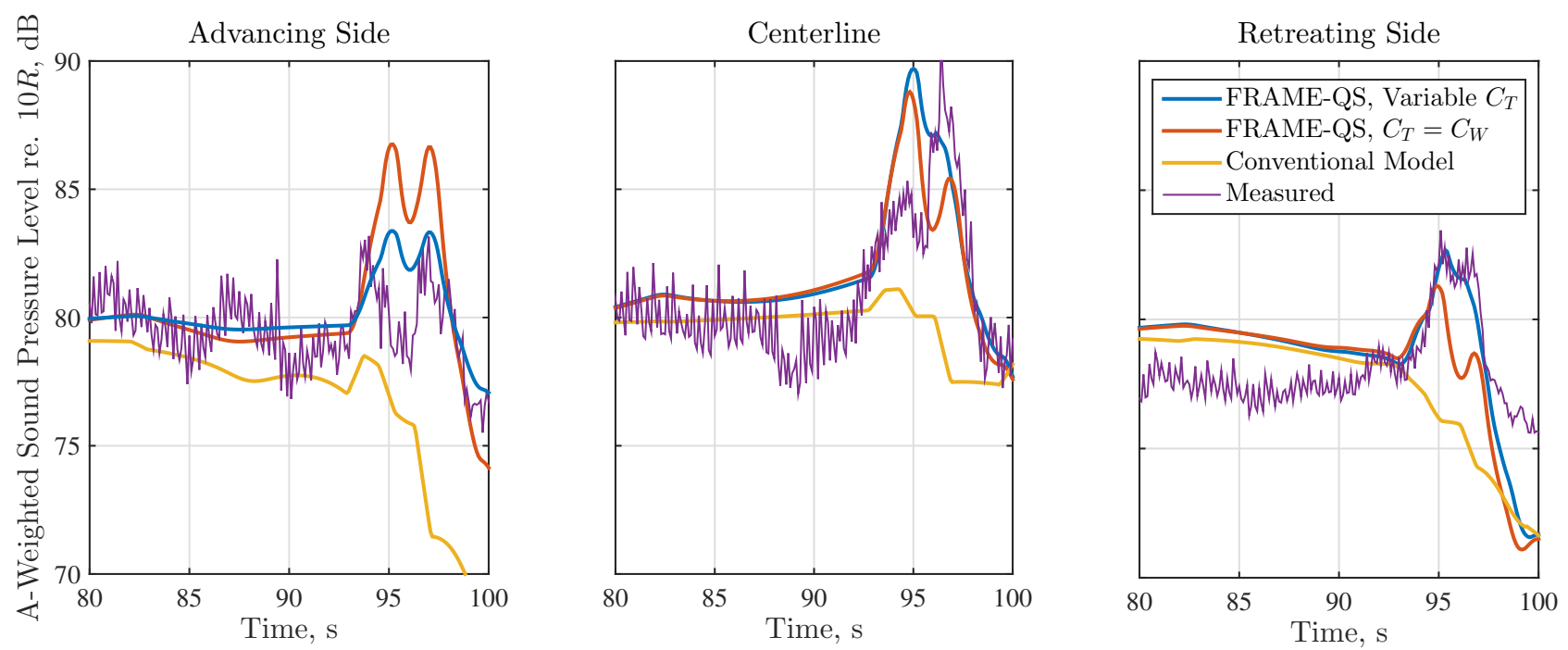

Fig. 7. A-weighted Sound Pressure Levels for the fast pitch up maneuver.

loading noise. However, on the advancing side, the constant load factor model overestimates the radiated noise. This is because the increased thrust results in an increased induced inflow through the rotor during the maneuver, changing the miss-distances for the BVI across the first quadrant of the rotor disk, attenuating those radiating towards the advancing side observer and amplifying those radiating towards the center and retreating side observers. This behavior is better captured in the variable $C_{T}$ FRAME-QS than in the constant thrust model. The "Conventional" model fails to model the transient increase in BVI because it neither changes the orientation of the sphere to radiate out-of-plane BVI to observers near the horizon, nor models the effective change in flight path angle, $\gamma_{e}$ due to the deceleration caused by the maneuver.

\section{Fast Roll Right from Level Flight}

In addition to the pitch up maneuver, a roll maneuver towards the advancing side of the rotor was examined. This maneuver was initiated at approximately the same location relative to the microphones as the pitch up maneuver. Figure 8 illustrates the measured lateral track, compared to the selected waypoints and predicted spline track. The predicted track agrees well with the measured trajectory, and once again results in a good estimate of the vehicle state. A plot of the measured and predicted roll angles for this track is shown in Figure 9.

A comparison of the three levels of modeling with the measured OASPL is shown for the fast roll right maneuver in Figure 10. The roll maneuver results in a significant increase in lower harmonic noise radiated towards the near-horizon observers as the helicopter banks through the turn, exposing the noise radiated below the rotor plane to the observers. The vari- 


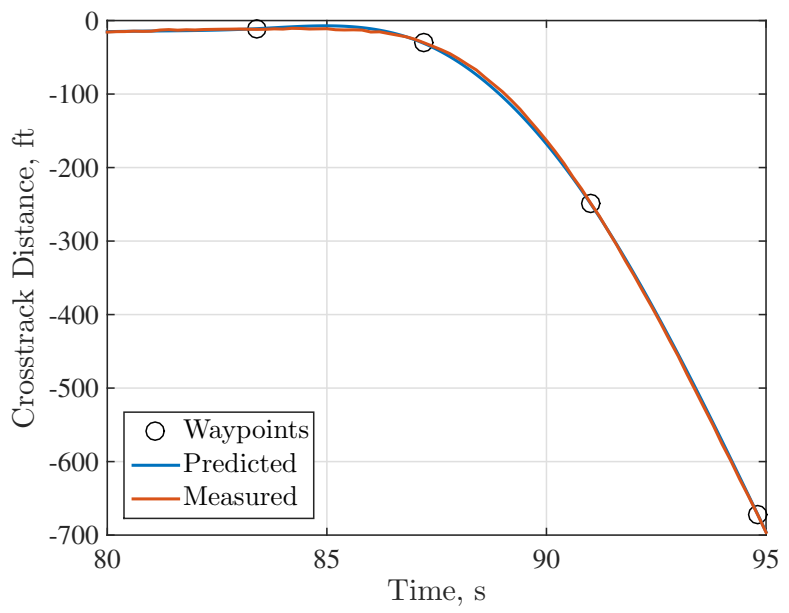

Fig. 8. Lateral trajectory during the roll maneuver.

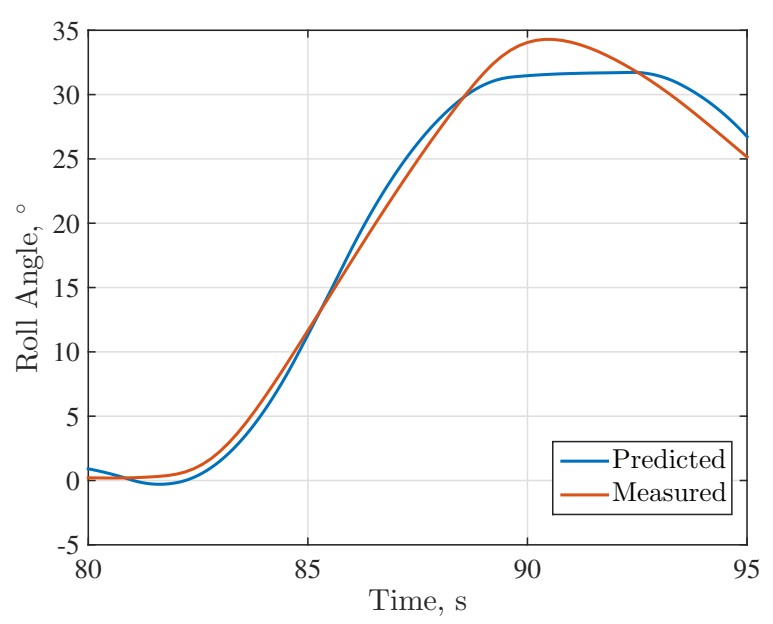

Fig. 9. Bank angle during the roll maneuver.

able load factor FRAME-QS model agrees well with the measured data, as the loading noise radiated below the banked rotor plane increases as the load factor of the helicopter increases to 1.4 during the roll maneuver. Conversely, the FRAME-QS model with constant load factor does not capture the transient increase in noise despite banking the noise radiation spheres in response to the acceleration. Likewise, the "Conventional" model, with constant load factor and unbanked spheres, shows even poorer agreement with the measured data.

Similar trends are seen for the A-weighted SPL comparisons shown in Figure 11. BVI noise amplitudes are less for the near-horizon observers during this maneuver than for the aggressive pitch up examined previously. The main rotor noise directed towards these observers responds to changes in load factor in a similar way as the lower harmonic noise does.

\section{Potential Limitations}

FRAME-QS was validated against near-horizon noise measurements in low wind conditions for the Bell 430 helicopter conducting both a pitch up maneuver and roll maneuver to- wards the advancing side with good agreement. However, the model makes several assumptions:

- The Q-SAM acoustic equivalence operates on the nondimensional governing parameters of the main rotor alone $\left(\mu, C_{T}, \alpha, M_{H}\right)$; the corresponding parameters of the tail rotor are assumed to vary with a one-to-one correspondance to variations in the main rotor operating state. Under some conditions, significant differences in the tail rotor operating condition could occur for the same main rotor operating condition, e.g. highly sideslipped flight.

- The drag of the fuselage was assumed insensitive to changes in the angles of attack and sideslip. For extremely aggressive maneuvers and helicopters with well streamlined fuselages, this assumption may be violated.

- The model neglects the impact of wind on the flight state of the vehicle, which could result in decreased accuracy when predicting the acoustic effects of transient maneuvers in windy conditions.

- The FRAME model does not yet include predictions for non-rotor-harmonic noise sources, such as rotor broadband noise and engine noise. This could result in the underprediction of noise levels for observers very close to, and directly underneath, the rotor.

Further validation with experimental data is required in order to detirmine the applicability of the model to circumstances in which these assumptions may be violated. If the effects of these assumptions are found to be significant, they can be relaxed in exchange for added complexity.

\section{CONCLUSION}

The FRAME-QS model developed in this paper was shown to accurately estimate the near-horizon noise radiation caused by transient maneuvers of the Bell 430 helicopter, using an approach that retains the speed and simplicity of existing source noise models used for mission planning which rely on databases of acoustic spheres. By applying models at several levels of fidelity to the estimation of maneuver noise, it was shown that:

- The Quasi-Static Acoustic Mapping (Q-SAM) concept is applicable to a range of transient maneuvers, in addition to longitudinal accelerations and steady turns.

- Accurate noise predictions must account for the significant changes in the rotor tip-path-plane orientation and load factor that occur during maneuvering flight.

- The Fundamental Rotor Acoustic Modeling from Experiments (FRAME) approach can be applied to pre-compute a source noise modeling database over the range of flight conditions, including load factors, that may be encountered during maneuvering flight. This is especially useful for those conditions that are difficult to measure using more empirical source noise characterization approaches. 

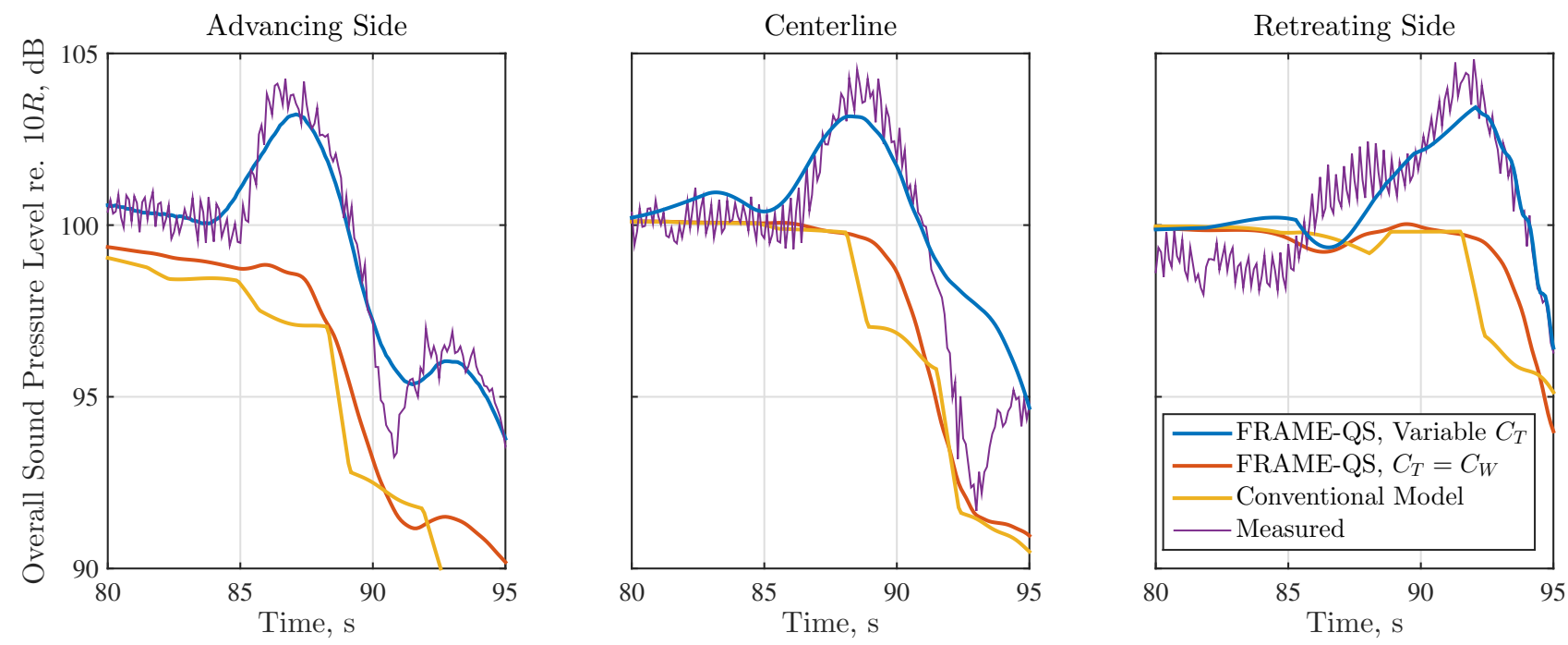

Fig. 10. Overall Sound Pressure Levels for the fast roll right maneuver.
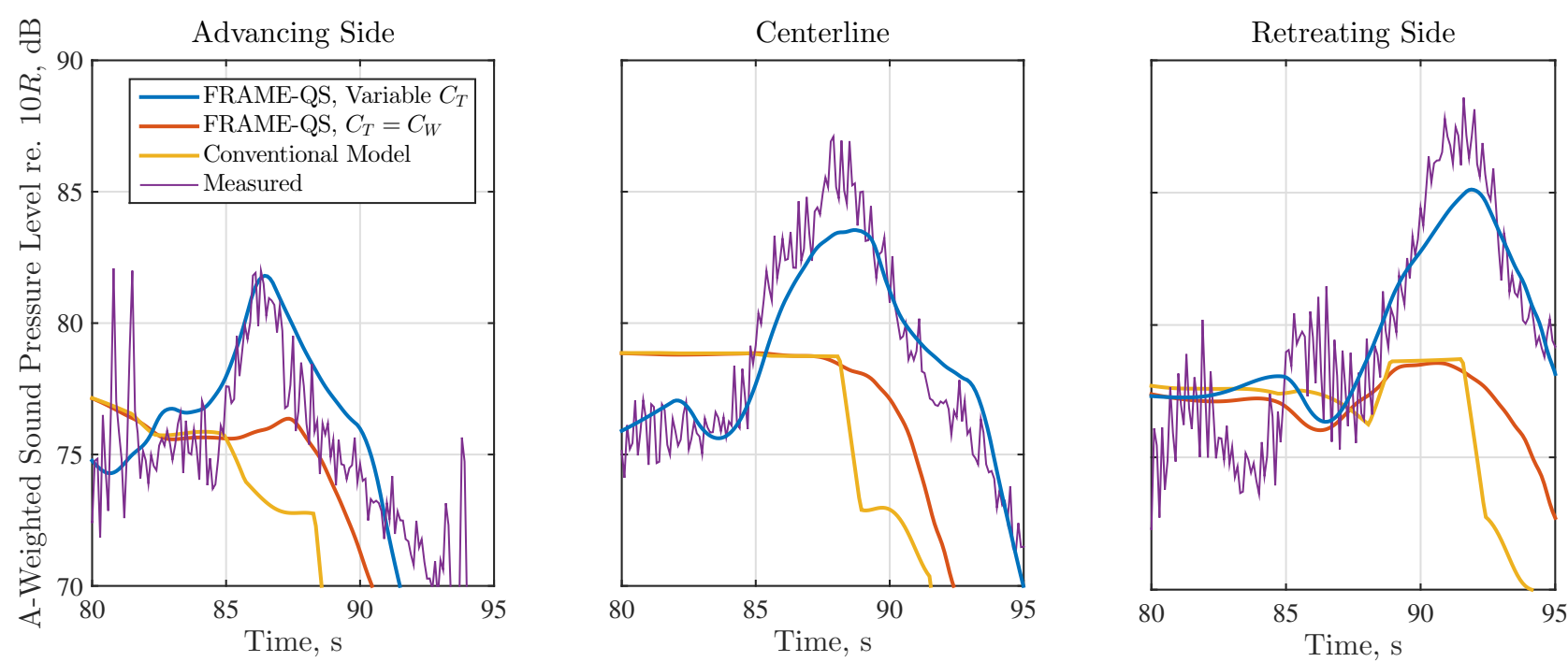

Fig. 11. A-weighted Sound Pressure Levels for the fast roll right maneuver.

\section{Applications}

The FRAME-QS source noise model developed in this paper can be incorporated into acoustic mission planning tools to estimate rotor harmonic noise radiation during steady and maneuvering flight. By generating a database of acoustic spheres for a wide range of flight conditions in advance and applying them under a quasi-static assumption later, the acoustic impact of a given flight trajectory can be rapidly assessed. This makes the model suitable for a number of applications, such as realtime noise estimation and for generating low-noise optimal mission profiles using a numerical optimizer.

\section{Author Contact}

Eric Greenwood, eric.greenwood@ nasa.gov

\section{ACKNOWLEDGEMENTS}

The authors would like to acknowledge the entire Bell 430 Maneuver Acoustics Flight Test team for providing the extensive data and analysis on which this paper is based, in particular Test Director Michael E. Watts, NASA Langley Research Center, and Bell Project Lead Royce Snider, Bell Helicopter Textron. Thanks to David A. Conner, U.S. Army, and Charles D. Smith, Analytical Mechanics Associates, for helpful discussions in the prepartion of this paper. In addition, a special thanks to Dr. Richard D. Sickenberger, whose research at the University of Maryland forms much of the basis for the model developed in this paper.

\section{REFERENCES}

${ }^{1}$ Sickenberger, R. D., Gopalan, G., and Schmitz, F. H., "Helicopter Near-Horizon Harmonic Noise Radiation due to Cyclic 
Pitch Control," American Helicopter Society 67th Annual Forum, May 2011.

${ }^{2}$ Lucas, M. J. and Marcolini, M. A., "Rotorcraft Noise Model," AHS Technical Specialists' Meeting for Rotorcraft Acoustics and Aerodynamics, October 1997.

${ }^{3}$ Conner, D. A. and Page, J. A., "A Tool for Low Noise Procedures Design and Community Noise Impact Assessment: The Rotorcraft Noise Model (RNM)," Heli Japan, 2002.

${ }^{4}$ Page, J. A., Wilmer, C., and Plotkin, K. J., "Rotorcraft Noise Model Technical Reference and User Manual," WR 08-04, Wyle, February 2008.

${ }^{5}$ Page, J. A., Wilmer, C., Schultz, T., Plotkin, K. J., and Czech, J., “Advanced Acoustic Model Technical Reference and User Manual," WP-1304, SERDP, May 2009.

${ }^{6}$ Spiegel, P., Buchholz, H., and Pott-Pollenske, M., "Highly Instrumented BO105 and EC135-FHS Aeroacoustic Flight Tests including Maneuver Flights," American Helicopter Society 61st Annual Forum, June 2005.

${ }^{7}$ Guntzer, F., Spiegel, P., and Lummer, M., "Genetic Optimizations of EC-135 Noise Abatement Flight Procedures using an Aeroacoustic Database," 35th European Rotorcraft Forum, September 2009.

${ }^{8}$ Schmitz, F. H., "Reduction of Blade-Vortex Interaction (BVI) Noise through X-Force Control," Journal of the American Helicopter Society, Vol. 43, (1), January 1998, pp. 14-24.

${ }^{9}$ Gopalan, G., Quasi-Static Acoustic Mapping of Helicopter
Blade-Vortex Interaction Noise, Ph.D. thesis, University of
Maryland, 2004 . Maryland, 2004.

${ }^{10}$ Sim, B. W., Schmitz, F. H., and Beasman, T., "Blade-Vortex Interaction (BVI) Noise of Helicopters Operating in Horizontal Wind Shear," American Helicopter Society 61st Annual Forum, May 2005.

${ }^{11}$ Schmitz, F. H., Greenwood, E., Sickenberger, R. D., Gopalan, G., Sim, B. W.-C., Conner, D. A., Moralez, E., and Decker, W., "Measurement and Characterization of Helicopter Noise in Steady-State and Maneuvering Flight," American Helicopter Society 63rd Annual Forum, May 2007.

${ }^{12}$ Greenwood, E., Schmitz, F. H., and Gopalan, G., "Helicopter External Noise Radiation in Turning Flight: Theory and Experiment," American Helicopter Society 63rd Annual Forum, May 2007.

${ }^{13}$ Greenwood, E. and Schmitz, F. H., "A Parameter Identification Method for Helicopter Noise Source Identification and Physics-Based Semi-Empirical Modeling," American Helicopter Society 66th Annual Forum, May 2010.

${ }^{14}$ Greenwood, E., Fundamental Rotorcraft Acoustic Modeling from Experiments (FRAME), Ph.D. thesis, University of Maryland, January 2011.
${ }^{15}$ Greenwood, E., Schmitz, F. H., and Sickenberger, R. D., "A Semi-Empirical Noise Modeling Method for Helicopter Maneuvering Flight Operations," American Helicopter Society 68th Annual Forum, May 2012.

${ }^{16}$ Sickenberger, R. D., Modeling Helicopter Near-Horizon Harmonic Noise due to Transient Maneuvers, Ph.D. thesis, University of Maryland, May 2013.

${ }^{17}$ Zefran, M. and Kumar, V., "Planning of Smooth Motions on SE(3)," IEEE International Conference on Robotics and Automation, Vol. 1, Apr 1996. doi: 10.1109/ROBOT.1996.503583

${ }^{18}$ Reinsch, C. H., "Smoothing by Spline Functions," $\mathrm{Nu}$ merische Mathematik, Vol. 10, 1967, pp. 177-183.

${ }^{19}$ Watts, M. E., Snider, R., Greenwood, E., and Baden, J., "Maneuver Acoustic Flight Test of the Bell 430 Helicopter," American Helicopter Society 68th Annual Forum, May 2012.

${ }^{20}$ Watts, M. E., Greenwood, E., Smith, C. D., Snider, R., and Conner, D. A., "Maneuver Acoustic Flight Test of the Bell 430 Helicopter Data Report,” TM2014-218266, NASA, 2014.

${ }^{21}$ Greenwood, E. and Sim, B. W., "The Effects of Crosswind Flight on Rotor Harmonic Noise Radiation," American Helicopter Society 69th Annual Forum, May 2013.

${ }^{22}$ Sibson, R., "A brief description of natural neighbour interpolation," Interpreting multivariate data, edited by V. Barnet, John Wiley \& Sons, 1981, pp. 21-36.

${ }^{23}$ Sickenberger, R. D. and Schmitz, F. H., "An Optics-Based Tip-Path Plane Tracking System for Rotorcraft Applications," AIAA Guidance, Navigation and Control Conference, Aug 2008. 